University of Wollongong

Research Online

Faculty of Engineering and Information

Faculty of Engineering and Information

Sciences - Papers: Part A

Sciences

$1-1-2011$

Location based services with personal area network for community and tourism applications

Montserrat Ros

University of Wollongong, montse@uow.edu.au

Matthew D'Souza

CSIRO ICT Centre, dsouza@itee.uq.edu.au

Adam Postula

University Of Queensland, adam@itee.uq.edu.au

I MacColl

Queensland University Of Technology

Follow this and additional works at: https://ro.uow.edu.au/eispapers

Part of the Engineering Commons, and the Science and Technology Studies Commons

Research Online is the open access institutional repository for the University of Wollongong. For further information contact the UOW Library: research-pubs@uow.edu.au 


\title{
Location based services with personal area network for community and tourism applications
}

\author{
Abstract \\ The Infopoint Explorer is an outdoor wireless personal area network for location-based services that \\ allows users to access locality information interactively using mobile computing devices such as PDAs \\ and smartphones. The Infopoint explorer was used to study the limitations imposed by inexpensive and \\ popular wireless technologies such as Bluetooth for outdoor location-based services. The Infopoint \\ Explorer functioned as a multimedia guidebook that allowed access to location-specific information using \\ wireless Infopoints. The Infopoints detect and attempt to transfer multimedia content to nearby mobile \\ computing devices using Bluetooth connectivity. An adaptive Bluetooth Inquiry Access Code algorithm \\ was developed to reduce the time taken for the Infopoint to detect nearby devices. The Infopoint was \\ deployed for a four-month trial.
}

\section{Keywords}

applications, tourism, location, community, network, area, personal, services

Disciplines

Engineering | Science and Technology Studies

\section{Publication Details}

M. Ros, M. D'Souza, A. Postula \& I. MacColl, "Location based services with personal area network for community and tourism applications," in IET International Communication Conference on Wireless Mobile and Computing (CCWMC 2011), 2011, pp. 432-437. 


\title{
Location Based Services with Personal Area Network for Community and Tourism Applications
}

\author{
Montserrat Ros ${ }^{1}$, Matthew D'Souza ${ }^{2}$, Adam Postula ${ }^{3}$, Ian MacColl ${ }^{4}$ \\ 1'montse_ros@uow.edu.au, School of ECTE, University of Wollongong, Wollongong, Australia \\ ${ }^{2}$ matthew.d'souza@csiro.au, CSIRO ICT Centre, Brisbane, Australia \\ 3adam@itee.uq.edu.au, School of ITEE, The University of Queensland, Brisbane, Australia \\ ${ }^{4}$ i.maccoll@qut.edu.au, Faculty of Science and Technology, Queensland University of Technology, Brisbane, \\ Australia
}

Keywords: Location-Based Services, Bluetooth Wireless Network, On-Demand Context-Aware Multimedia

\begin{abstract}
The Infopoint Explorer is an outdoor wireless personal area network for location-based services that allows users to access locality information interactively using mobile computing devices such as PDAs and smartphones. The Infopoint explorer was used to study the limitations imposed by inexpensive and popular wireless technologies such as Bluetooth for outdoor location-based services. The Infopoint Explorer functioned as a multimedia guidebook that allowed access to location-specific information using wireless Infopoints. The Infopoints detect and attempt to transfer multimedia content to nearby mobile computing devices using Bluetooth connectivity. An adaptive Bluetooth Inquiry Access Code algorithm was developed to reduce the time taken for the Infopoint to detect nearby devices. The Infopoint was deployed for a four-month trial.
\end{abstract}

\section{Introduction}

The widespread usage of sophisticated mobile computing devices such as smartphones and Personal Digital Assistants (PDAs) has created new ways of facilitating interactive location-based services and social networking applications for communities and tourism sites. With features such as cameras, touchscreen displays, motion sensors and audio outputs, mobiles are powerful platforms for innovative interactive systems. These features have made mobile devices attractive for use with location-based services and mobile social networking that require multimedia content access. Most advanced location-based services using mobile devices require a wireless network such as Wireless LAN, cellular 3G or GSM/GPRS to facilitate interaction with users. The drawback of using such networks is that an extensive infrastructure, user subscription and mobile devices with specific capabilities are required to access the provided location-based services. Personal Area Networks can overcome such drawbacks by providing a simpler, localised and inexpensive wireless network infrastructure that can facilitate wireless multimedia connections with different mobiles. This can be advantageous for museums, historical or any other sites that want to offer users interactive location-based services without installing an extensive infrastructure or requiring user-specific mobile computing device capabilities or prior subscription.

Our aim was to study the challenges of deploying a personal area network for an outdoor tourism promotion location based service. These challenges can be summarised as: capability to provide low latency transactions; high capacity, sufficient data transfer bandwidth; universal usage on different mobiles and the use of low 'green' power infrastructure. We developed the interactive Infopoint Explorer to provide location-based services for multimedia tourism and community applications for the Kelvin Grove Urban Village (KGUV) [1] in Brisbane, Australia, a historical tourist trail in Fremantle, Australia and the 2009 Brisbane Festival.

The Infopoint Explorer functions as a multimedia guidebook and enables users to access information when within range of a monument or artifact, pervasively. The Infopoint Explorer consists of a network of wireless Bluetooth enabled nodes known as Infopoints located at sites of interest, such as monuments. Each Infopoint continually scans for nearby Bluetooth-enabled mobiles and once a mobile is discovered, the Infopoint initiates a connection to transfer multimedia content to the user's mobile.

The advantage of the Infopoint Explorer was that it was able to provide a low latency and universal mechanism of sending multimedia content to different mobile smartphones. We also manipulated the 


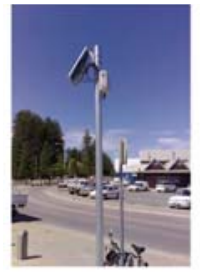

A) Infopoint in Fremantle

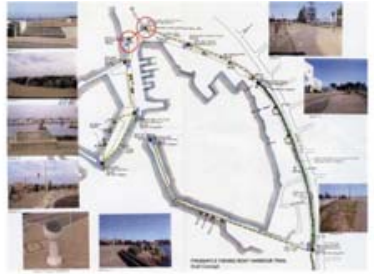

B) Proposed Tourist Trail along Fremantle Whart

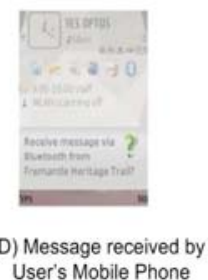

User's Mobile Phone

C) Example Content - Gift Voucher

Figure 1: Fremantle Infopoint Tourist Trail

Bluetooth inquiry process to facilitate shorter scanning periods by implementing an adaptive inquiry access code algorithm.

We conducted an extensive evaluation of the Infopoint Explorer, over a period of four months. The initial Infopoint Explorer consisted of a network of three Infopoint placed at different sites. The Infopoint recorded events such as mobile discovery, file transfer connectivity and acceptance.

In Section 2, we present a review of related work. Sections 3, 4 and 5 discuss the implementation of the Infopoint Explorer. Further work and conclusions are given in sections 6 and 7 .

\section{Related Work}

Location-based services include localised information retrieval, games and map-based way-finding systems. Various solutions exist to implement location-based services. We examine and compare existing research relating to location-based services applications with an emphasis on multimedia guidebooks.

A multimedia guidebook is a location-based service that provides users with access to information related to their current locations. There are two forms of multimedia guidebooks. One involves information being transferred to the mobile on request and the second stores the information locally on the mobile for use by the user on request or at a particular time. Examples of guidebooks include the Exploratorium [7], [6] and the Cyberguide [2]. The Exploratorium guidebook uses infrared beacons, $802.11 \mathrm{~b}$ wireless network and PDAs. When a user with a PDA comes within range of an infrared beacon, a Uniform Resource Locator (URL) number is transmitted by infrared to the user's PDA. A Hypertext Transfer

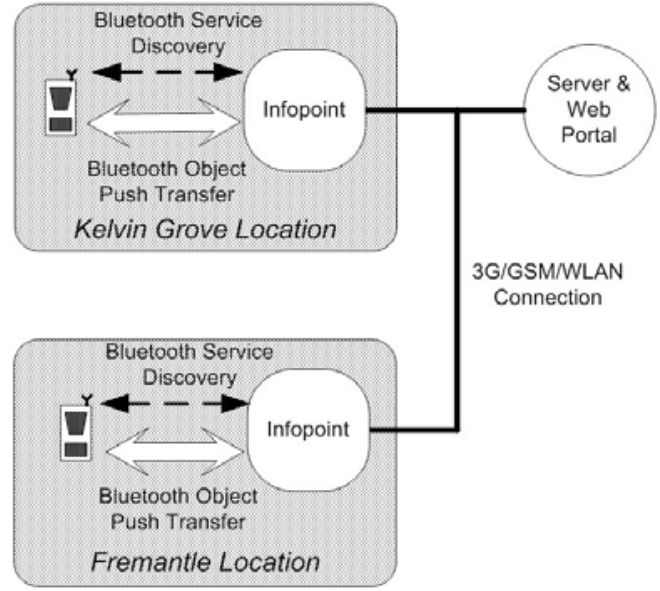

Figure 2: Infopoint Explorer Infrastructure

Protocol (HTTP) link is then used to request the desired information using an $802.11 \mathrm{~b}$ WLAN connection.

One of the disadvantages of this approach is the use of infrared technology for interaction. The user must be within line of sight of the infrared beacon. Also, the use of $802.11 \mathrm{~b}$ WLAN connections restricts the types of mobile computing devices that can be used. Our Infopoint Explorer uses Bluetooth wireless communications which is available on a wide range of different mobile platforms because it is more robust than infrared. Bluetooth communications does not have line of sight restrictions as infrared does and it also supports legacy protocols such as the Object Exchange Protocol (OBEX) developed for data files transfers via infrared.

PlaceSense by Nguyen et al [8] is an example of a pervasive Bluetooth location based service. PlaceSense requires Bluetooth enabled agents to be situated in the environment and mobiles with a client application installed. Like the Infopoint, a Bluetooth enabled agent scans for nearby Bluetooth devices that are running the PlaceSense application. Messages regarding to the services offered by a particular agents are then sent to the user. The user has the option to accept or reject the service. Unlike the Infopoint, PlaceSense requires an application programmed to be installed on the mobile. This does limit the number of users that can use services, as different mobile models would require a version of the PlaceSense application.

\section{Infopoint Explorer Overview}

The Infopoint Explorer uses the Bluetooth protocol to discover and communicate with nearby mobiles as shown in Figure 1 and Figure 2. Bluetooth was chosen as the wireless communications protocol due to its 


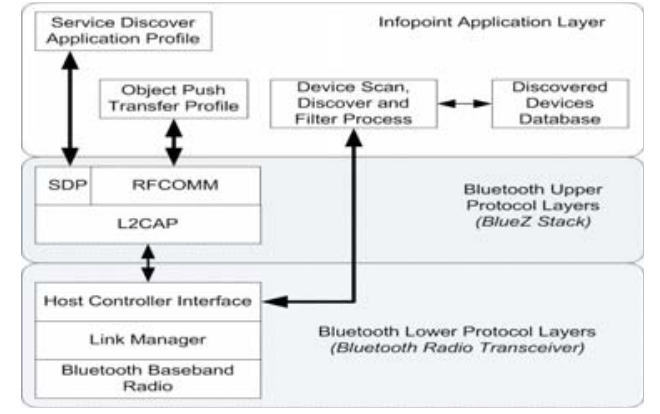

Figure 3: Infopoint use of Bluetooth Protocol Stack

prevalence on mobiles. This fact, coupled with the nature of Bluetooth communications lacking the requirement for line-of-sight, means users can retrieve location-aware information without additional software or hardware.

The Infopoints used two of Bluetooth's information transfer profiles, Service Discovery Application Profile (SDAP) and Object Push Profile (OPP) [4], that are supported by most mobiles. Figure 3 shows how the Bluetooth communications protocol stack is used by the Infopoints for discovery and file transfer. The SDAP is used to discover nearby mobile computing devices that are capable of receiving multimedia content files. The OPP is used to transfer information items from the Infopoint to the user's mobile.

Figure 4 shows the discovery and transfer process. The discovery process initially uses the Host Control Interface (HCI) inquiry mechanism and service discovery protocol to scan for nearby Bluetoothenabled mobiles, with a five second timeout. Once the scan period is completed, discovered devices are filtered using the Bluetooth class identifier. The Bluetooth class identifier describes the type of device, such as smartphone, laptop, printer, etc. The Infopoint only initiates a transfer to devices that are classed as mobiles or PDA devices.

The Infopoint then attempts to transfer the multimedia content file, via an OPP connection, to each detected mobile. Each mobile's Bluetooth address is compared to a database of previously detected mobiles. If the mobile's Bluetooth address is in the database, then the control process skips that particular mobile. This is to ensure that a user does not receive the multimedia content multiple times when within scanning range of the Infopoint. The database is completely refreshed after the control process has finished transferring the file to each mobile.

The Infopoint was able to manipulate the Bluetooth inquiry scan process to reduce the time required for

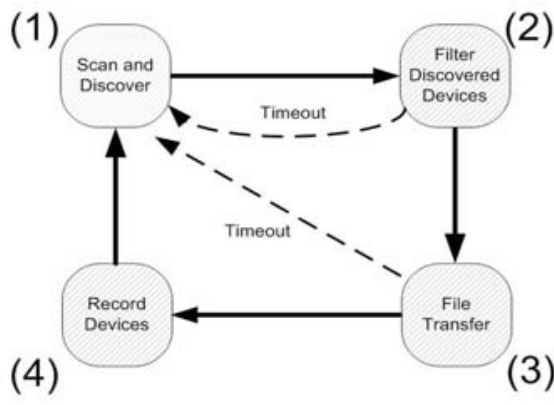

Figure 4: Infopoint Discovery and Transfer Process

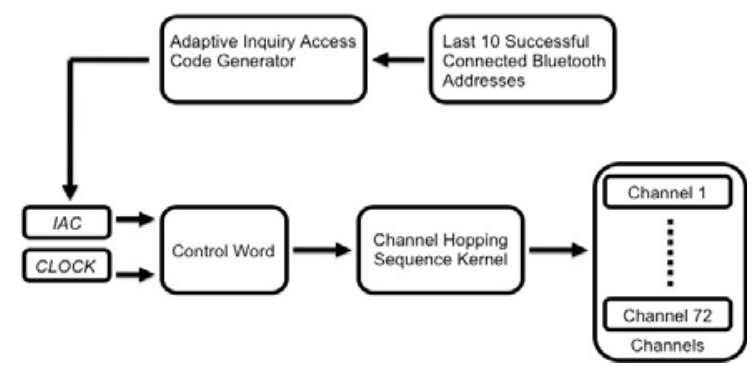

Figure 5: Adaptive Inquiry Access Code Generator

detecting nearby devices. The Bluetooth Inquiry process can take a lengthy period of time $(\sim 20 \mathrm{~s})$ to detect nearby devices [3]. This is due to the Frequency Hopping Spread Spectrum (FHSS) modulation scheme used by the Bluetooth physical layer. The Bluetooth operating frequency band is divided into 79 channels [4] and so a typical Bluetooth inquiry process will scan a channel for devices for 625us. If no devices are discovered, another channel to scan is then selected according to a channel hopping sequence. The Bluetooth channel hopping sequence is based on a persuado-random sequence that is created using an inquiry access code and the Bluetooth device's clock. This generates a unique persuado-random channel hopping sequence.

The channel hopping sequence kernel can be seen in Figure 5 and is dependent on the control word. The control word was derived using an inquiry access code and the clock value/offset of the device. The Inquiry Access Code (IAC) is typically assigned the General Inquiry Access Code (GIAC) for normal Bluetooth scanning. During normal transactions, the IAC is assigned the last 28 bits of the Bluetooth device address.

\subsection{Adaptive Inquiry Access Code}

The Infopoint used an adaptive Inquiry Access Code to scan for nearby mobiles. If the channel hopping 


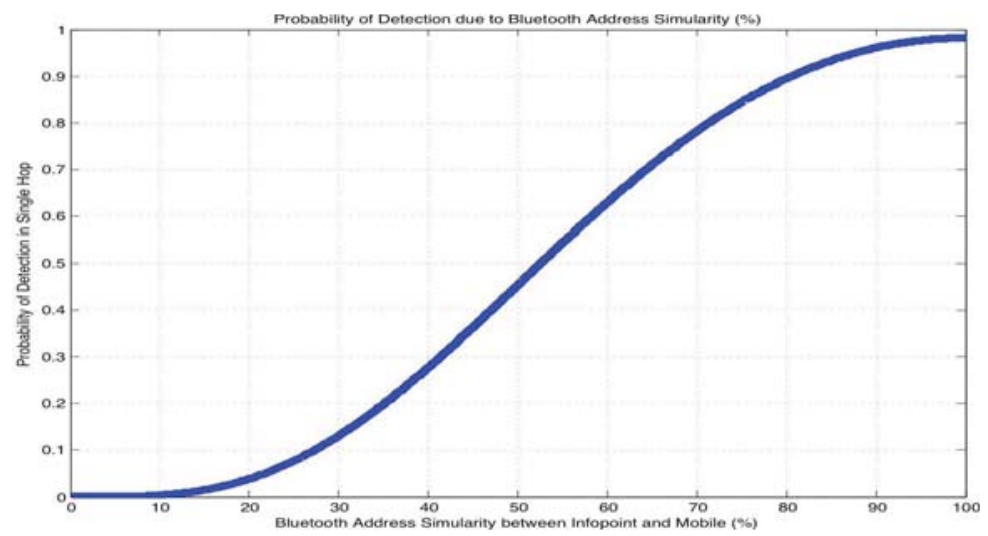

Figure 6: Estimated Probability of Detection due to Bluetooth Address Similarity.

sequence of both the Infopoint and mobile are bitwise similar, then the scanning time can be reduced. This is because the devices can discover each other using fewer channel hops. In order to manipulate the channel hopping sequence, an adaptive IAC mechanism was developed. The adaptive IAC was calculated using the average of Bluetooth device addresses of the ten last known successfully connected mobiles. Equation 1 is used to calculate the bit array of the Adaptive IAC. Each 4-bit section of the AIAC is calculated using the corresponding 4 bits of the last ten connected mobiles' Bluetooth addresses.

$$
A I A C=\left[\frac{1}{N} \sum_{n=0}^{N} U_{n}^{3.0}, \frac{1}{N} \sum_{n=0}^{N} L_{n}^{11 . .8}, \frac{1}{N} \sum_{n=0}^{N} L_{n}^{7.4}, \frac{1}{N} \sum_{n=0}^{N} L_{n}^{3.0}\right]
$$

Where:

AIAC $=$ Adaptive Inquiry Access Code $\mathrm{N}=10$ (Number of Bluetooth Addresses),

$\mathrm{U}=\mathrm{UAP}$ section of the Bluetooth Address,

$\mathrm{L}=\mathrm{LAP}$ section of the Bluetooth Address,

The estimated performance of the adaptive IAC was modelled as a Poisson probability distribution function. Poisson probability and cumulative distribution is commonly used to model Bluetooth network performance [11] in terms of scanning time and probability of detection or connection. Figure 6 shows the estimated detection probability versus similarity between the Infopoint and mobile Bluetooth address. As the Infopoint and mobile Bluetooth addresses become similar, the channel hopping sequence becomes the same for both devices and hence the detection probability increases. As shown in Figure 7 as the probability improves, the scanning time will decrease. It was estimated that the average scanning time was reduced from $5 \mathrm{~s}$ to $2 \mathrm{~s}$ using the adaptive IAC algorithm.

\section{Implementation}

The open source Linux Bluez Bluetooth stack software [5] is used to facilitate the Bluetooth connectivity to the mobile. Used Bluetooth protocol layers include the Service Discovery Protocol (SDP), L2CAP and HCI Bluetooth stack layers [4]. Functions are also provided to add or remove services from the SDP Database. The SDP was used to discover nearby compatible Bluetooth mobiles and the Object Push Profile was used to transfer the multimedia content to the user's mobile.

Catering for multiple types of Bluetooth enable mobiles was challenging due to the differing implementations of the Service Discovery Application and Object Push Profiles. To ensure that most types of devices could be catered for, timeout functions were implemented to ensure that it took less than five seconds to transfer a multimedia content file to a user's mobile. It was found that maintaining short scan periods and connection times were essential to the usability of the Infopoint.

\section{Deployment and User Experience}

A solar-powered Infopoint was deployed in Fremantle, Australia, and was extensively evaluated over a fourmonth period. The Infopoint Explorer also consisted of Infopoints deployed at two other sites in Brisbane, Australia, and Christchurch, New Zealand. It is intended to expand the Infopoint Explorer Network to four Infopoints in the Kelvin Grove Urban Village [1] in the near future. Four Infopoints were also deployed for the duration of the 2009 Brisbane Festival [9] throughout the West End historical walking trail. Early indications from trials showed that the slow access speed was a substantial issue for users. 


\begin{tabular}{|c|c|c|c|c|c|c|c|}
\hline & $\begin{array}{l}\text { Detected } \\
\text { Devices } \\
\text { (Including Non- } \\
\text { Mobiles) }\end{array}$ & $\begin{array}{l}\text { Connections } \\
\text { Attempted } \\
\text { (Only to } \\
\text { Mobiles) }\end{array}$ & $\begin{array}{l}\text { Connection } \\
\text { Accepted but } \\
\text { Transfer Error } \\
\text { Occurred }\end{array}$ & $\begin{array}{l}\text { Connections } \\
\text { Accepted }\end{array}$ & $\begin{array}{l}\text { Connections } \\
\text { Refused }\end{array}$ & $\begin{array}{l}\text { Connection } \\
\text { Ignored }\end{array}$ & $\begin{array}{l}\text { Connection } \\
\text { Error }\end{array}$ \\
\hline Total & 21051 & 8094 & 623 & 309 & 1313 & 4888 & 961 \\
\hline $\begin{array}{l}\text { Total } \\
(\%)\end{array}$ & - & $\begin{array}{l}38 \% \text { (of } \\
\text { devices) }\end{array}$ & $\begin{array}{l}8 \% \text { (of } \\
\text { attempted } \\
\text { connections) }\end{array}$ & $\begin{array}{l}4 \% \text { (of } \\
\text { attempted } \\
\text { connections) }\end{array}$ & $\begin{array}{l}16 \% \text { (of } \\
\text { attempted } \\
\text { connections) }\end{array}$ & $\begin{array}{l}60 \% \text { (of } \\
\text { attempted } \\
\text { connections) }\end{array}$ & $\begin{array}{l}12 \% \text { (of } \\
\text { attempted } \\
\text { connections) }\end{array}$ \\
\hline
\end{tabular}

Table 1: Total Connections Attempted

Table 1 shows the total number of connections attempted during the four-month trial in Fremantle. During the trial period, over 21000 devices including non-mobiles were detected. Over 8000 connections to mobiles were attempted. The total number of connections accepted was 309 (4\%) but 4888 (60\%) connections were ignored with 1313 (16\%) connections being refused. Factors that could have contributed to this are:

- Mobile Bluetooth notifications were disabled.

- Bluetooth notification not noticed while within range of the infopoint

- Bluetooth file transfer notification detected but:

- Not accepted before timing out.

- Unwilling to participate so notification ignored.

- Unaware of how to participate so notification not accepted.

\section{Further Work}

Further work on the Infopoint Explorer will involve expanding its capabilities and applying the Infopoint to other applications. One problem with the prototype implementation is that it is restricted to a single
Bluetooth module and, as a result, the Infopoint alternates between discovery and transfer (that is, between SADP and OPP). A further problem to be addressed is the limited bandwidth available under Bluetooth that manifests in two ways. Firstly, multimedia files tend to be large and must be compressed to transfer in a reasonable amount of time from a user perspective. Experimentally, we found that files needed to be under $100 \mathrm{~KB}$ to transfer quickly enough for average users. Secondly, mobiles in a context such as a tourist trail may be moving fairly quickly and so may move out of range in less than the time needed for discovery and transfer, particularly if there are a large number of mobiles in the area.

Wireless LAN or Bluetooth 3.0 capability will also be added to future Infopoints to increase bandwidth to provide "richer" location-based content for WLAN or Bluetooth 3.0 capable mobiles and to increase the coverage area. The Bluetooth 3.0 protocol high file transfer rates [10] would be advantageous for interacting with the Infopoint. Enabling user interaction with the Infopoint using visual, audio and motion sensitive inputs will also be explored. Future Infopoint Explorer enhancements will enable user to more directly interact with the by uploading comments and multimedia content as a "trail" for other users to

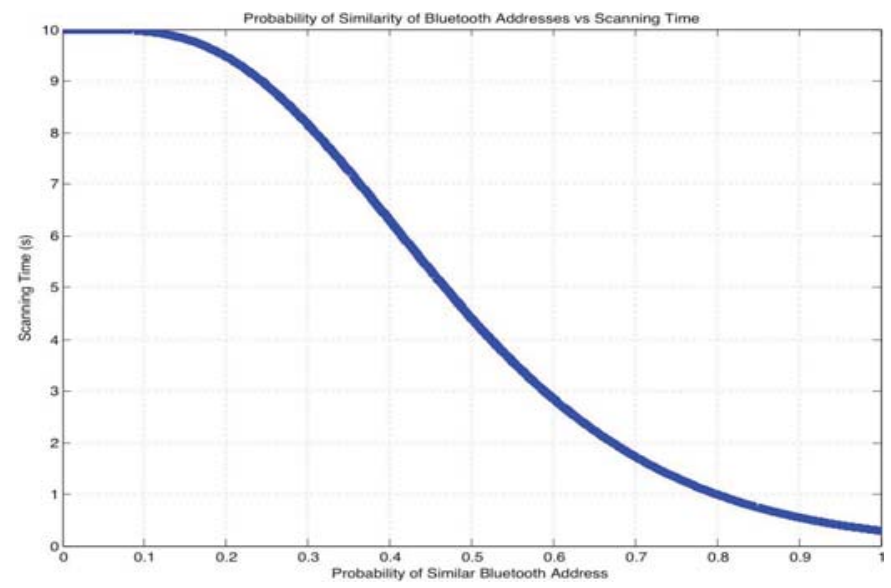

Figure 7: Estimated Scanning Time Durations due to Probability of Similar Addresses. 
follow and access. An evaluation workshop also suggested the possibility of using the Infopoint for civic activism.

\section{Conclusion}

The Infopoint Explorer is a location-based service that enabled users to access information about their immediate surroundings using mobile computing devices. Users view images, listen to audio and read textual information at specific locations through Infopoints embedded in the local environment. Infopoints are dispersed at sites of interest and offer nearby users, multimedia content that can be accessed on their personal mobiles without any requirement for additional software or hardware or pre-existing registration.

The Infopoint detected and transferred multimedia content to nearby mobiles. Bluetooth communications was used as the transport for this location-based service. We found that Bluetooth while was advantageous in detecting and transferring multimedia content to mobiles, there were time delay issues were encountered. These delays were caused when creating Bluetooth connections, transferring multimedia content to different models of mobiles and prolonged scanning periods. In order to reduce the scanning period, an adaptive Bluetooth inquiry access code algorithm was created. The adaptive Bluetooth inquiry access code, manipulated the Bluetooth device hopping sequence so that both Infopoint and mobile could be detect each other quicker. It was estimated that the scanning time decreased from $5 \mathrm{~s}$ to $2 \mathrm{~s}$.

\section{Acknowledgements}

We would like to thank Ingrid Richardson, John McMullan, Ian Clough, Mark Billinghurst and Gang Wang for their assistance in supporting the Infopoint Explorer trial. We also thank the Fremantle City Council for allowing us to trial the Infopoint Explorer in a public space in Fremantle, Australia.

\section{References}

[1] A. B. Author, C. D. Author. "Title of the article", The Journal, volume, pp. 110-120, (2000).

[1] "Kelvin Grove Urban Village", http://www.kgurbanvillage.com.au/.

[2] G. D. Abowd, C. G. Atkeson, J. Hong, S. Long, R. Kooper, and M. Pinkerton, "Cyberguide: a mobile context-aware tour guide," Wirel. Netw., vol. 3, pp. 421-433, 1997.

[3] N. Baker, "ZigBee and Bluetooth strengths and weaknesses for industrial applications," Computing \& Control Engineering Journal, vol. 16, pp. 20-25, 2005.

[4] Bluetooth SIG, " Bluetooth Specification Documents", www.bluetooth.org/spec.

[5] BlueZ-Project, "BlueZ - Official Linux Bluetooth Protocol Stack." www.bluez.org.

[6] A. M. Haneef, A. Ganz, "Mobile agent based network access for mobile electronic guidebooks," presented at Mobility and Wireless Access Workshop, 2002.

[7] S. Hsi, "The Electronic Guidebook: A study of user experiences using mobile web content in a museum setting," presented at IEEE International Workshop on Wireless and Mobile Technologies in Education, 2002.

[8] T. Nguyen; S. W. Loke; T. Torabi and H. Lu, "PlaceSense: A Tool for Sensing Communities," in 4th International Symposium on Wireless Pervasive Computing, 2009. ISWPC 2009.

[9] Brisbane Festival 2009, www.brisbanefestival.com.au

[10] Bluetooth SIG , "Bluetooth 3.0 + HS Specification", Vol. 0, April 21, 2009.

[11] T. Lin, Y. Liu, Y. Tseng, "An improved packet collision analysis for multi-Bluetooth piconets considering frequency-hopping guard time effect", Selected Areas in Communications, IEEE Journal on, vol.22, no.10, pp. 2087- 2094, Dec. 2004 